\section{Avances del decenio de educación ambiental para el desarrollo sustentable (deds) 2005-2014 en la Universidad Juárez Autónoma de Tabasco}

\author{
Eduardo S. López Hernández * \\ Silvia Cappello García * \\ Ana Rosa Rodríguez Luna **
}

\section{ARTICULO ORIGINAL}

Fecha de recibido: 06 Mayo de 2009

Fecha de aceptación: 01 Julio de 2009

\section{DIRECCIÓN PARA RECIBIR CORRESPONDENCIA}

Dr. Eduardo S. López-Hernández

División Académica de Ciencias Biológicas UJAT, Km 0.5 carr. Villahermosa-Cárdenas s/n

Col. Bosques de Saloya

86039, Villahermosa, Tabasco.

eduardos.lopezh@gmail.com

* Cuerpo Académico de Ecología y Conservación de ecosistemas vegetales. División Académica de Ciencias Biológicas. Universidad Juárez Autónoma de Tabasco

** El Colegio de la Frontera Sur-Unidad Villahermosa.

Educación Ambiental

Eduardo S. López Hernández

Silvia Cappello García *

Ana Rosa Rodríguez Luna *

HORIZONTE SANITARIO

45
La década de la educación para el desarrollo sustentable (DEDS) de Naciones Unidas posiblemente es una oportunidad única para las instituciones y la ciudadanía para cooperar en este tema, y remarca la responsabilidad que tienen los gobiernos de trabajar en este nuevo paradigma. Ha sido la línea de educación ambiental para el desarrollo sustentable del Cuerpo Académico de Ecología y Conservación de Ecosistemas Vegetales de la División Académica de Ciencias Biológicas los que han ejecutado tales acciones, con experiencia mayor a dos décadas con influencia nacional, regional e internacional. Como objetivo perseguimos evidenciar los avances al momento en el decenio en aspectos como educación formal, no formal y profesionalización de educadores ambientales, capacitación, vinculación y extensión a estudiantes y pueblos indígenas. Partimos de una revisión bibliográfica extensiva, planeación en diseños curriculares, para programas de asignatura en licenciatura, maestría y educación continua. Se elaboraron materiales didácticos para capacitación de grupos en el medio rural, y formal con variopintos temas sobre problemas globales. Se hizo una sistematización de resultados de nuestros avances. Se entrega una relación del desarrollo de la dimensión ambiental en el plano de la reforma curricular del plan flexible y el impulso del programa ambiental para la desarrollo sustentable, aportaciones institucionales de la Universidad Juárez Autónoma de Tabasco (UJAT) al Decenio 2005-2014, una de las 16 mejores instituciones de educación superior en México que tiene acreditados al $100 \%$ sus programas educativos en materia ambiental. 
Palabras Clave - Educación Ambiental, Decenio, Universidad pública, Educación superior

\section{SUMMARY}

The Decade of the Education for Sustainable Development (DEDS) of United Nations possibly is the only opportunity for the institutions and the citizenship to cooperate in this topic, and notices the responsibility that the governments have of being employed at this new paradigm. It has been the line of environmental education for the sustainable development of the Academic Staff on Ecology and Conservation of Plant Ecosystems at the Academic Division of Biological Sciences those that have executed such actions, with experience bigger than two decades with national, regional and international influence.

Since we prosecute aim to demonstrate the advances to the moment in the decade in aspects like formal, not formal education and professionalization of environmental educators, training, links and extension to students and indigenous peoples. We depart from a bibliographical extensive review, planning in curriculum developments, for programs of subject in licentiate, mastery and continue education. Didactic materials were elaborated for training group in the rural and formal way by many different topics on global problems.

There was done a systematizing result of our advances. There is delivered a relation of the development of the environmental dimension in the plane of the reform curricular of the flexible plan and the impulse of the environmental program for sustainable development, institutional contributions of the Universidad Juarez Autonoma de Tabasco (UJAT) to the Decade 2005-2014, one of 16 better institutions of higher education in
Mexico that has her educational programs credited to $100 \%$ in environmental matter.

Key words: Environmental education, Decade, Public University, Higher Education 


\section{INTRODUCCIÓN}

El factor humano tiene unas características que lo ubican por encima de otras especies, entre ellas su capacidad intelectual y la utilización de ella en la confección, fabricación y empleo de múltiples objetos y herramientas para la modificación del medio, la generación de la cultura y el desarrollo de sus sociedades.

Diversos factores que han colocado al hombre en el planeta como el principal provocador de la crisis ambiental. En la actualidad miles de millones de personas viven en la pobreza absoluta, un número importante de ellas sin posibilidades de cubrir sus necesidades básicas de alimentación, vestido y vivienda.

Los gobiernos han estimulado la sobreexplotación de los recursos naturales todos. Una tendencia de mal reparto de la riqueza, ha repercutido en las decisiones del manejo y transformación de los bienes naturales.

Por ejemplo, en los países tropicales por décadas han estado obligados a destruir las selvas para la búsqueda de espacios productivos y hacerlos viables para su uso agrícola. Muchas de estos sitios carecían de condiciones idóneas, y sin embargo, estimularon el progresivo avance de la erosión, pérdida de suelos y fertilidad, como la disminución de las importantes reservas de semillas del bosque, es decir, una degradación de los procesos ecológicos básicos.

La inteligencia humana, tanto como la capacidad de nuestra especie por modificar el entorno, siempre con base en nuestra supervivencia, ha pasado a lo largo de la historia de la tierra, desde su aparición, por una serie de eventos que le han posibilitado aprender sobre los recursos naturales, sobre su utilidad y además descubrir sus cualidades para mejorar sus condiciones de vida. En ese orden de ideas, la explicación del significado de la intervención del hombre en la naturaleza desde épocas remotas, y con respecto a la época contemporánea en el siglo XXI, definen las circunstancias de las modificaciones que sufre el medio natural, y por ende, los problemas globales planetarios. López-Hernández (2003)

La problemática ambiental no es un fenómeno reciente y complejo, los problemas ambientales existen desde que la especie humana apareció en el horizonte evolutivo y comenzó a aprovechar los recursos naturales y a transformar el medio en su beneficio. En la actualidad se relaciona con el estilo de desarrollo y la falta de respeto a la diversidad que nos rodea, con ello los problemas conexos con los que hay que trabajar se establecen en:

1. Biológico: nuevos conocimiento, concienciación y participación - acción

2. Ambiental: hacia la revalorización y cambio actitudinal

3. Cultural (étnica): respeto, solidaridad, hermandad, tolerancia

4. Social y económica: mediante una repartición equitativa de la riqueza, democracia, cambio social crítico y científico y

5. Conservación: superando la visión clásica por la de espacios de utilidad múltiple

Por todo lo anterior desde 1992 a la fecha, hacia un futuro con desarrollo sostenible las conspicuas metas de evaluación y solución son:

A. Los problemas ambientales globales: el impacto del hombre en la naturaleza, y los problemas del desarrollo. 
B. Principales problemas nacionales y regionales a partir de sus causas y efectos

C. Acuerdos y acciones para propiciar su solución y la prevención de su avance con la Educación Ambiental.

D. Objetivos para la aplicación de la Educación Ambiental.

E. Desarrollo Sostenible.

F. Participación de la sociedad

\section{ANTECEDENTES}

La Conferencia de la Organización de las Naciones Unidas sobre «Medio Ambiente Humano» en Estocolmo (Suecia) en 1972. En este evento se planteaba ya, la gravedad de la situación ambiental mundial por los problemas documentados científicamente en el planeta, determinando una tendencia que debiera tratarse con carácter global. Las conclusiones obtenidas encauzaron una nueva convocatoria. Subrayaba que temas como medio ambiente y desarrollo se debían reconocer como partes de un todo. Una muestra de estos planteamientos se registraron el documento El Desarrollo Sustentable: Transformación productiva, equidad y medio Ambiente (Santiago de Chile, 1991). Importantes también fueron la organización de la UNICED y la resolución de la Asamblea General que anunció e invito a los estados miembros a promover la participación amplia de la comunidad científica, la industria, los sindicatos, las asociaciones en general y con especial referencia a las sociedad civil pretendiendo enriquecer las deliberaciones y la divulgación de los resultados y su integración en políticas concretas.

La primera reunión preparatoria de la UNICED realizada en Vancouver, Canadá (1990) reunió

Vol. 8 no2

Mayo- Agosto 2009

HORIZONTE SANITARIO

48 unas 115 organizaciones civiles de unos 40 países para estudiar su papel en el proceso. Se decidió promover una amplia participación de todos los sectores de la sociedad civil en todo el mundo. Esos primeros esfuerzos permitieron la interacción de los gobiernos con los grupos civiles organizados para el enriquecimiento del proceso pero esto se diluyó y no se alcanzó ese propósito, pero dio pautas para establecer una comunicación intensa entre la sociedad civil por medio de un proceso paralelo al de los gobiernos. La ONU y algunos organizadores, brindaron todas las facilidades para los trabajos de las organizaciones no gubernamentales, a través del Comité Internacional de Facilitación, durante las PrepCom (Nairobi: agosto de 1990 y Ginebra: marzo de 1992). Finalmente en Brasil (junio de 1992) tuvieron participación dentro del Foro Global de los Centros Focales Regionales, las propuestas se difundieron en el documento del Foro Internacional, Compromisos para el Futuro y Proceso de Elaboración de los Tratados Alternativos. (López-Hernández, 2003)

Se desarrolló una ardua labor por unificar criterios, redactar y revisar los documentos, para obtener una propuesta compatible con los enfoques tan diversos de regiones, países, formas de desarrollo y situaciones sociales. En Río de Janeiro en 1.992, se elaboraron por fin las versiones finales de los tratados, mismos que fueron finalmente firmados. Los resultados en el papel, reflejan el difícil trabajo de conciliación, más no de negociación y representan un compromiso formal para aplicarlos. Hay que destacar que el término «tratado» es utilizado para representar el carácter alternativo pretendido por estos documentos ante los acuerdos oficiales firmados.

En diciembre de 2002, en la resolución 57/254 de la Asamblea General de las Naciones Unidas, se 
declaró el Decenio de la Educación para el Desarrollo Sustentable. La UNESCO tiene a su cargo la preparación de un Plan de Aplicación Internacional y fue distinguida como la organización para realizarlo de 2005 a 2014. El DEADS guarda una fuerte la relación con las metas del Milenio para el Desarrollo y los proyectos educativos en marcha son:

El Marco de Acción de Dakar emergido del Foro Mundial sobre la Educación (2000), y el Decenio de las Naciones Unidas para la Alfabetización, programas que definen la calidad de la educación como un prerrequisito de la educación para el desarrollo sustentable en todos los niveles y modalidades.

La educación para el desarrollo sustentable aunque se ha tomado como un concepto dinámico y polémico, ha empleado indivisos aspectos de la conciencia pública, la educación y la capacitación, para intentar conducirnos al desarrollo de conocimientos, y sus vínculos entre desarrollo sustentable las habilidades, valores, competencias y perspectivas. Así, distinguimos que la educación para el desarrollo sustentable posee puntos fundamentales como los que citamos:

- Promoción y mejoramiento de la educación básica. La calidad de la educación básica debe mejorar el enfoque sobre la difusión del conocimiento, habilidades, valores y perspectivas a través de la vida, que fomente y apoye a los ciudadanos a llevar una vida sustentable

- Desarrollar la comprensión y la conciencia pública acerca de la sustentabilidad. Para avanzar hacia sociedades más sustentables se requiere ciudadanos informados y consumidores conocedores.
- Capacitación. El desarrollo de programas especializados de capacitación se ha identificado como un componente crítico para asegurar que todos los sectores de la fuerza de trabajo tengan el conocimiento y las habilidades necesarias para desempeñar su trabajo de una manera sustentable.

No existirá un modelo universal de la educación para el desarrollo sustentable, mientras no haya un acuerdo general sobre el concepto, habrá diferencias matizadas de acuerdo con los contextos locales, las prioridades y los enfoques, por lo que cada nación deberá definir sus propias prioridades y acciones.

La planeación de programas y actividades del Decenio presenta algunos temas clave que son de carácter crítico para la implementación del proyecto. Entre los más importantes hay que destacar la base material para este cambio de paradigma como son la biodiversidad, gestión y conservación de agua dulce, conservación del ambiente, transformación rural, promoción de la salud, producción y consumo sustentable, derechos humanos, paz y entendimiento internacional, y los temas transversales de alivio de la pobreza e igualdad de género.

Los temas anteriores, de hecho ya habían sido planteados en la Universidad Juárez Autónoma de Tabasco como base de su posicionamiento ante este decenio. (López-Hernández y Priego, 2007). 


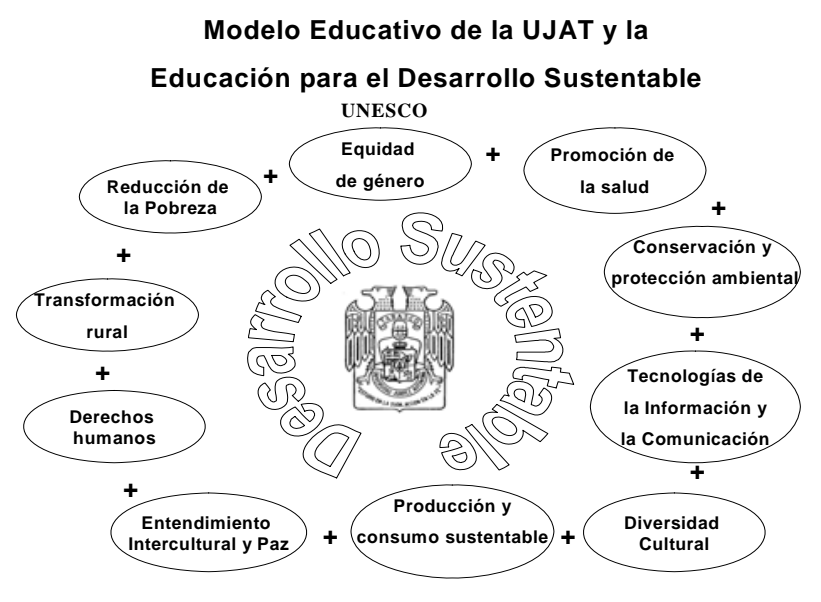

Por lo anterior este Decenio, es parte de la política institucional centrada en el alumno y en el modelo educativo de la UJAT en torno a que en la educación superior, está considerada como una estrategia central para el desarrollo sustentable.

La Década para el Desarrollo Sustentable de Naciones Unidas es una oportunidad única para comprometer a las instituciones y a la ciudadanía en general a cooperar con este tema. La UNESCO remarca la responsabilidad que tienen los gobiernos de trabajar por la Década para el Desarrollo Sustentable. Sin embargo, este esfuerzo se debe dar en dos sentidos: desde las bases (organizaciones de vecinos, asociaciones civiles, etc.) hacia arriba y desde el gobierno, instituciones, etc. a las bases. Achinelli (2005).

Para lograr un efectivo paso desde la educación ambiental a la EDS es importante tener en cuenta los valores actuales de la sociedad (relación Naturaleza-Humanidad-Sociedad) y la incorporación no sólo de los temas ambientales sino también de los políticos, sociales y económicos. Por lo tanto, la EDS suma a la dimensión ambiental cuestiones tales como:

- Abordaje del concepto de desarrollo sustentable entendido desde una perspectiva interdisciplinaria.
- Pensamiento crítico para la resolución de problemas.

- Métodos múltiples de enseñanza (aplicando distintas pedagogías)

- Conexión entre los temas globales y locales.

Otros aspectos muy importantes lo establecen el respeto y promoción de la diversidad cultural, lingüística y ecológica, recuperando la historia indígena. Asimismo, se señala que las sociedades humanas son las que deben determinar su destino y que, «el conocimiento acumulado y producido socialmente, no debe ser patentado o monopolizado».

Al respecto del enfoque educativo, se presenta la sólida base de la formación de una conciencia local y planetaria, una ética sobre todas las formas de vida, ubicando al individuo en relación a la naturaleza y el universo desde una perspectiva histórica y social.

Para ello indica, que deberá promoverse la cooperación y diálogo entre individuos e instituciones, así como democratizar y usar los medios masivos de comunicación. Se considera que el trabajo debe abarcar la educación formal, la no formal y la informal, tanto como la capacitación.

El resultado de Río de Janeiro, estableció 22 acciones a realizar. Entre ellas algunas son lineamientos competentes al trabajo educativo, tales como:

1. Considerar a la educación como base de sociedades sostenibles,

2. Procurar la creación de conocimientos, políticas y metodología educativa, 
3. Promover la corresponsabilidad de hombres y mujeres y erradicar el racismo y la discriminación sexual,

4. Exaltar la diversidad cultural, y la autodeterminación de los pueblos,

5. Fortalecer las organizaciones y movimientos sociales y transformar los hábitos consumistas y los sistemas que los sustentan.

Establece también que se tratarán de aplicar los principios de este tratado de acuerdo con las realidades locales. Otras corresponden a acciones más concretas como son difundir información sobre conocimientos populares y tecnologías adecuadas, promover la creación de Consejos Populares de Acción Ecológica y Gestión Ambiental, crear redes nacionales y mundiales sobre temas socioeconómicos y ecológicos, elaborar documentos para ser utilizados en la red formal de enseñanza y en programas educativos de movimientos sociales y exigir a los gobiernos que destinen un porcentaje significativo de sus presupuestos para educación y medio ambiente.

Por su carácter global, el tratado aspira a involucrar a organizaciones y movimientos sociales, ONG, educadores, medios de comunicación, científicos, gobiernos nacionales y locales, empresarios y comunidades en general, todos ellos responsables y dispuestos a procurar un desarrollo sostenible.

En consecuencia, los propósitos de esta colaboración son:

- Informar acerca de los avances en el trabajo desarrollado para este decenio en aspectos de la educación formal, la no formal y la profesionalización de educadores ambientales
-Dar a conocer la capacitación, vinculación y extensión de la labor universitaria hacia diferentes comunidades humanas y los pueblos indígenas.

\section{MATERIALES Y MÉTODOS}

Esta consistió de una revisión bibliográfica extensiva en educación ambiental, desarrollo sustentable, investigación participativa, etc. Complementada con la planeación de las acciones en el marco del DEAS (SEMARNAT, 2005), y el PRAUJAT (López-Hernández y Priego, 2007) en lo relativo al diseño curricular, la vinculación y la educación continua (diplomado de educación ambiental). Se realizó de acuerdo al modelo educativo de la UJAT el diseño curricular y programas de asignaturas de estudios en los niveles licenciatura, maestría y educación continua (desarrollo comunitario, salud, agroecología, entre otros). Como complemento se elaboraron materiales didácticos para la capacitación de grupos humanos en el medio rural y formal sobre aspectos relacionados con las bases educativas en programas de educación ambiental, organización social para la sustentabilidad y sobre los problemas globales: agua, calentamiento global, conservación de biodiversidad, carta de la tierra y de aspectos productivos en el área de la agroecología, y otros temas. Se finalizó con una sistematización de resultados e informes de los avances alcanzados.

\section{RESULTADOS}

La información que hemos aportado para la integración de un Informe de País, con especificidad estatal para integrar un reporte sobre los avances del Decenio de Educación para el Desarrollo Sostenible 2005-2014, de la Universidad Juárez Autónoma de Tabasco (UJAT), una Universidad pública en el área 
Mesoamericana, que ha acreditado al $100 \%$ sus programas educativos en materia ambiental y se encuentra entre las 16 mejores Instituciones de Educación Superior de México, cuya aportación al desarrollo de la educación ambiental se centra en información crucial para este propósito. Ha sido la línea de Educación Ambiental para el desarrollo sustentable del Cuerpo Académico de Ecología y Conservación de ecosistemas tropicales y la División Académica de Ciencias Biológicas los que han proyectado, ejecutado y afianzado tales acciones, con una experiencias ahora de más de dos décadas con influencia en la región sursureste y a nivel nacional e internacional.

Las contribuciones institucionales tiene que ver con las 10 Áreas Temáticas prioritarias establecidas por la Organización de las Naciones Unidas (ONU), las que a saber son las siguientes: reducción de la pobreza, equidad de género, promoción de la salud, conservación y protección ambiental, transformación rural, derechos humanos, entendimiento intercultural y paz, producción y consumo sustentable, diversidad cultural y tecnologías de la información.
Entre los resultados trascendentes para la UJAT están el programa estatal de educación ambiental, capacitación y comunicación para la sustentabilidad, La postura de nuestra institución de educación superior ante el DEADS, las recomendaciones del programa ambiental adheridas al curriculum flexible, el programa de educación continúa y vinculación, las propuestas de la gestión ambiental y la incorporación de estudiantes en el servicio social comunitario de este programa en la universidad, entre otros.

La tabla I refleja las actividades, participantes y público meta, en ambientes no formales mediante una relación de resultados de los procesos educativos estructurados y actuados en sendos cursos-talleres en torno a la educación ambiental y la sustentabilidad, que fueron aplicados por el personal académico en el marco de extensión universidad-sociedad a través de sus programas de investigación científica, servicio social comunitario, y educación continua vinculación extensión. Todos los anteriores en el marco del PRAUJAT. (López et al. 2009)

Tabla I.

ACTIVIDADES DE EDUCACIÓN AMBIENTAL PARA LA SUSTENTABILIDAD

EN LA UJAT A PARTIR DE ENERO DE 2005

\begin{tabular}{|c|c|c|c|c|}
\hline ACTIVIDAD & PARTICIPANTES & DESTINATARIOS & REALIZACIÓN & RESULTADO \\
\hline $\begin{array}{l}\text { Curso taller } \\
\text { composta con } \\
\text { mujeres indígenas }\end{array}$ & $\begin{array}{l}\text { Universidad } \\
\text { Juárez Autónoma } \\
\text { de Tabasco } \\
\text { El Colegio de } \\
\text { Investigadores de } \\
\text { Tabasco } \\
\text { Fundación Mundo } \\
\text { Sustentable A. C. }\end{array}$ & $\begin{array}{l}\text { Mujeres jóvenes, } \\
\text { Madres de familia y } \\
\text { señoras de la tercera } \\
\text { edad }\end{array}$ & 2006 & $\begin{array}{l}\text { Formación de un grupo de } 30 \\
\text { señoras capacitadas } \\
\text { Creación de las bases de una } \\
\text { MYPIME de composta } \\
\text { Diseño curricular de educación } \\
\text { ambiental }\end{array}$ \\
\hline
\end{tabular}

Vol. 8 no2

Mayo- Agosto 2009

HORIZONTE SANITARIO 


\begin{tabular}{|c|c|c|c|c|}
\hline $\begin{array}{l}\text { Curso taller PET } \\
\text { con } \\
\text { indígenas }\end{array}$ & $\begin{array}{l}\text { Universidad } \\
\text { Juárez Autónoma } \\
\text { de Tabasco } \\
\text { El Colegio de } \\
\text { Investigadores de } \\
\text { Tabasco } \\
\text { Fundación Mundo } \\
\text { Sustentable A. C. }\end{array}$ & $\begin{array}{l}\text { Jóvenes y jovencitas } \\
\text { indígenas chontales } \\
\text { de } 14 \text { a } 21 \text { años }\end{array}$ & 2006 & $\begin{array}{l}\text { Formación de un grupo de } 20 \\
\text { jóvenes y jovencitas chontales } \\
\text { capacitadas } \\
\text { Creación de las bases de una } \\
\text { MYPIME de gestión de PET } \\
\text { Diseño curricular de educación } \\
\text { ambiental }\end{array}$ \\
\hline $\begin{array}{lr}\text { Curso } & \text { taller } \\
\text { manejo adecuado } \\
\text { de los } \\
\text { contenedores de } \\
\text { separación de RSU }\end{array}$ & $\begin{array}{l}\text { Universidad } \\
\text { Juárez Autónoma } \\
\text { de Tabasco } \\
\text { El Colegio de } \\
\text { Investigadores de } \\
\text { Tabasco } \\
\text { Fundación Mundo } \\
\text { Sustentable A. C. }\end{array}$ & $\begin{array}{l}\text { Profesores, } \\
\text { Estudiantes, y } \\
\text { Personal } \\
\text { administrativo de las } \\
\text { Divisiones } \\
\text { Académicas de } \\
\text { Educación y Artes } \\
\text { Ciencias Sociales y } \\
\text { Humanidades, } \\
\text { Ciencias de la Salud, } \\
\text { Ciencias } \\
\text { Agropecuarias, } \\
\text { Ciencias Económico } \\
\text { Administrativas, } \\
\text { Ciencias Biológicas }\end{array}$ & 2006 & $\begin{array}{l}\text { Manuel de Manejo de contendores } \\
\text { de separación } \\
\text { Jornada de Conferencias } \\
\text { Preparación práctica } \\
\text { Dotación de contenedores a las } \\
\text { divisiones académicas } \\
\text { colaboradoras } \\
\text { Reforestación } \\
\text { Limpieza de los campus }\end{array}$ \\
\hline $\begin{array}{l}\text { Curso taller de } \\
\text { creación artística } \\
\text { carta de la tierra }\end{array}$ & $\begin{array}{l}\text { Universidad } \\
\text { Juárez Autónoma } \\
\text { de Tabasco } \\
\text { El Colegio de } \\
\text { Investigadores de } \\
\text { Tabasco } \\
\text { Fundación Mundo } \\
\text { Sustentable A. C. }\end{array}$ & $\begin{array}{l}\text { Niños chontales de } 8 \\
\text { a } 14 \text { años }\end{array}$ & 2006 & $\begin{array}{l}\text { Niños chontales } \\
\text { dibujaron loa } 16 \text { principios } \\
\text { Pintado de un mural } \\
\text { Adaptación al idioma chontal los } \\
16 \text { principios de carta de la tierra } \\
\text { Exposición de dibujos en el Museo } \\
\text { de Historia Natural } \\
\text { Participación en el Fórum }\end{array}$ \\
\hline
\end{tabular}




\begin{tabular}{|c|c|c|c|c|}
\hline & & & & $\begin{array}{l}\text { Universal de la Culturas Monterrey, } \\
\text { N. L. } \\
\text { Edición de un libro }\end{array}$ \\
\hline $\begin{array}{l}\text { Programa } \\
\text { ambiental para el } \\
\text { desarrollo } \\
\text { sustentable de la } \\
\text { UJAT }\end{array}$ & $\begin{array}{l}\text { Universidad } \\
\text { Juárez Autónoma } \\
\text { de Tabasco } \\
\text { El Colegio de } \\
\text { Investigadores de } \\
\text { Tabasco } \\
\text { Promotora } \\
\text { Ambiental SAB de } \\
\text { CV } \\
\text { H. Ayuntamiento } \\
\text { de Centro }\end{array}$ & $\begin{array}{l}\text { Profesores, } \\
\text { Estudiantes, y } \\
\text { Personal } \\
\text { administrativo de las } \\
\text { Divisiones } \\
\text { Académicas de } \\
\text { Educación y Artes } \\
\text { Ciencias Sociales y } \\
\text { Humanidades, } \\
\text { Ciencias de la Salud, } \\
\text { Ciencias } \\
\text { Agropecuarias, } \\
\text { Ciencias Económico } \\
\text { Administrativas, } \\
\text { Ciencias Biológicas }\end{array}$ & 2005 & $\begin{array}{l}\text { Desarrollo del Programa Ambiental } \\
\text { Institucional por medio de Talleres } \\
\text { de Investigación Acción } \\
\text { Participativa con Estudiantes, } \\
\text { Profesores, Sindicatos y } \\
\text { Sociedades de Alumnos } \\
\text { Documento y plan operativo } \\
\text { Informe a la Rectora }\end{array}$ \\
\hline $\begin{array}{l}\text { Jornadas por un } \\
\text { campus digno y } \\
\text { limpio primera fase }\end{array}$ & $\begin{array}{l}\text { Universidad } \\
\text { Juárez Autónoma } \\
\text { de Tabasco } \\
\text { El Colegio de } \\
\text { Investigadores de } \\
\text { Tabasco } \\
\text { Promotora } \\
\text { Ambiental SAB de } \\
\text { CV } \\
\text { H. Ayuntamiento } \\
\text { de Centro }\end{array}$ & $\begin{array}{l}\text { Profesores, } \\
\text { Estudiantes, y } \\
\text { Personal } \\
\text { administrativo de las } \\
\text { Divisiones } \\
\text { Académicas de } \\
\text { Educación y Artes } \\
\text { Ciencias Sociales y } \\
\text { Humanidades, } \\
\text { Ciencias de la Salud, } \\
\text { Ciencias } \\
\text { Agropecuarias, } \\
\text { Ciencias Económico } \\
\text { Administrativas, } \\
\text { Ciencias Biológicas }\end{array}$ & 2005 & $\begin{array}{l}\text { Ciclo de Conferencias } \\
\text { Comunicación de los Resultados } \\
\text { del PRAUJAT } \\
\text { Capacitación sobre el manejo de } \\
\text { residuos sólidos del Programa } \\
\text { Ambiental Institucional } \\
\text { Talleres de Investigación Acción } \\
\text { Participativa con Estudiantes, } \\
\text { Profesores, Sindicatos } \\
\text { Sociedades de Alumnos } \\
\text { Documento y plan operativo }\end{array}$ \\
\hline Jornadas por & Universidad & Profesores, & 2006 & Conferencias \\
\hline
\end{tabular}




\begin{tabular}{|c|c|c|c|c|}
\hline $\begin{array}{ll}\text { campus } & \text { digno y } \\
\text { limpio } & \text { segunda } \\
\text { fase } & \end{array}$ & $\begin{array}{l}\text { Juárez Autónoma } \\
\text { de Tabasco } \\
\text { El Colegio de } \\
\text { Investigadores de } \\
\text { Tabasco } \\
\text { Promotora } \\
\text { Ambiental SAB de } \\
\text { CV }\end{array}$ & $\begin{array}{l}\text { Estudiantes, y } \\
\text { Personal } \\
\text { administrativo de las } \\
\text { Divisiones } \\
\text { Académicas de } \\
\text { Educación y Artes } \\
\text { Ciencias Sociales y } \\
\text { Humanidades, } \\
\text { Ciencias de la Salud, } \\
\text { Ciencias } \\
\text { Agropecuarias, } \\
\text { Ciencias Económico } \\
\text { Administrativas, } \\
\text { Ciencias Biológicas }\end{array}$ & & $\begin{array}{l}\text { Comunicación de los Resultados } \\
\text { del PRAUJAT } \\
\text { Actualización de problemas socio- } \\
\text { ambientales de estudiantes de la } \\
\text { UJAT } \\
\text { Problemática ambiental y de } \\
\text { gestión de los planteles } \\
\text { Matriz de problemas } \\
\text { Matriz de posibles soluciones } \\
\text { Organigrama funcional del } \\
\text { PRAUJAT } \\
\text { Manual de Manejo de contendores } \\
\text { de separación } \\
\text { Reforestación } \\
\text { Limpieza de los campus } \\
\text { Levantamiento de encuestas }\end{array}$ \\
\hline $\begin{array}{l}\text { Consejo ciudadano } \\
\text { de educación } \\
\text { ambiental de } \\
\text { Tabasco }\end{array}$ & $\begin{array}{l}\text { SEMARNAT } \\
\text { SEDESPA } \\
\text { El Colegio de } \\
\text { Investigadores de } \\
\text { Tabasco } \\
\text { Fundación Mundo } \\
\text { Sustentable A. C. } \\
\text { Promotora } \\
\text { Ambiental SAB de } \\
\text { CV } \\
\text { Universidad } \\
\text { Juárez Autónoma } \\
\text { de Tabasco }\end{array}$ & & $2000-2008$ & $\begin{array}{l}\text { Organización de Foros estatales y } \\
\text { regionales de educación } \\
\text { ambiental, } \\
\text { Elaboración de Agenda ambiental } \\
\text { Colaboración en la política de } \\
\text { educación capacitación y } \\
\text { comunicación para } \\
\text { sustentabilidad de Tabasco }\end{array}$ \\
\hline
\end{tabular}




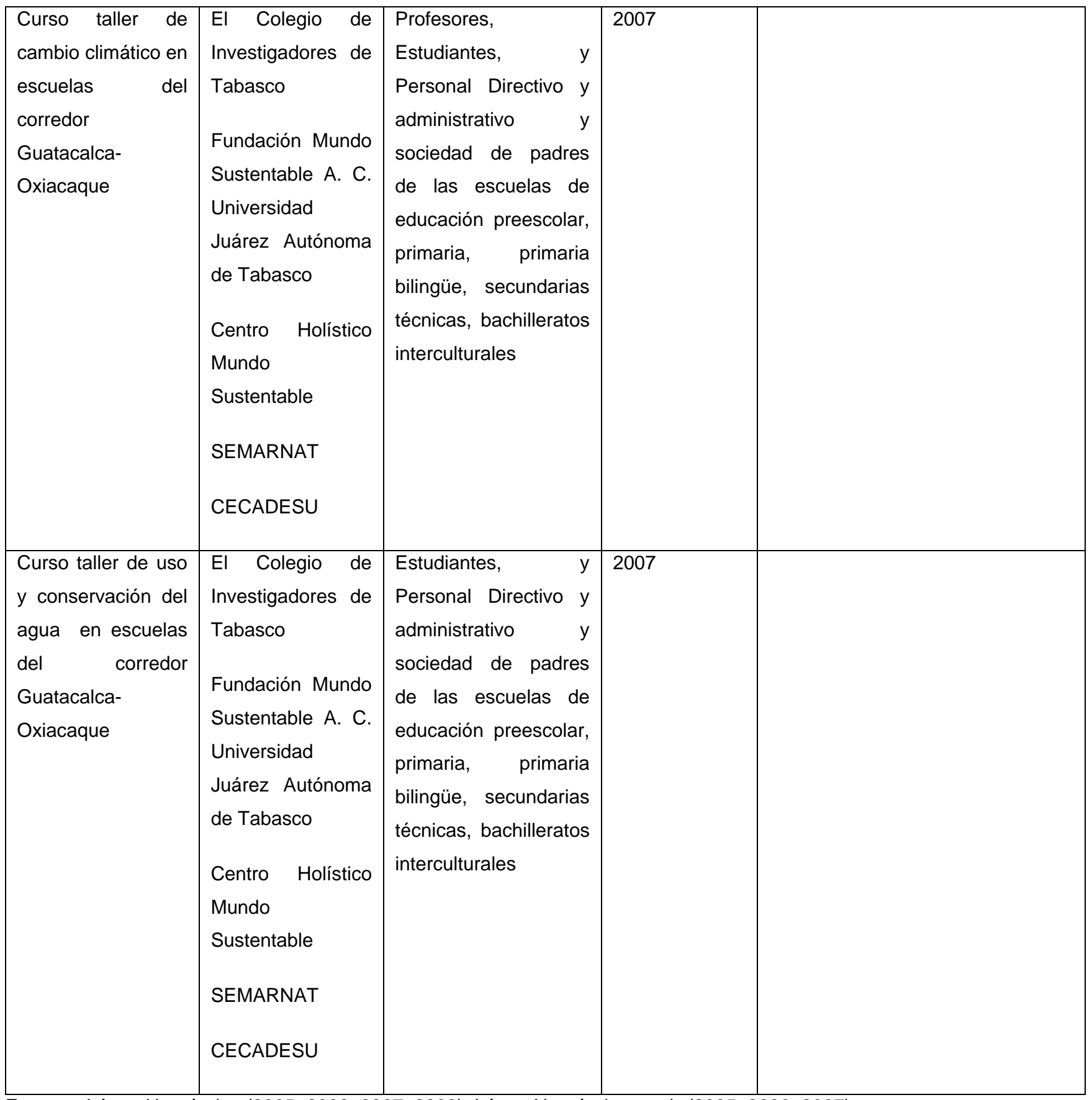

Fuentes: López-Hernández (2005, 2006, 2007, 2009); López-Hernández e. al. (2005, 2006, 2007).

La tabla II corresponde a las actividades, participantes y público meta, en ambientes formales (universidad, educación continua, posgrado, escuelas primarias, etc.) mediante una relación de resultados de educación ambiental para el desarrollo sustentabilidad del personal

Vol. 8 no2

Mayo- Agosto 2009

HORIZONTE SANITARIO

56 académico en el marco de actuación de investigación científica, diseño curricular, formación y profesionalización de recursos humanos y en la oferta educativa de la UJAT. (López et al. 2009). 
Tabla II.

PROGRAMAS O ACTIVIDADES DE EDUCACIÓN AMBIENTAL PARA LA SUSTENTABILIDAD A NIVEL FORMAL

EN LA UJAT A PARTIR DE ENERO DE 2005

\begin{tabular}{|c|c|c|c|c|}
\hline $\begin{array}{l}\text { NOMBRE DEL } \\
\text { PROGRAMA O } \\
\text { ACTIVIDAD }\end{array}$ & $\begin{array}{l}\text { INSTITUCIONES } \\
\text { PARTICIPANTES }\end{array}$ & POBLACIÓN DESTINATARIA & $\begin{array}{l}\text { PERIODO DE } \\
\text { REALIZACIÓN }\end{array}$ & $\begin{array}{l}\text { RESULTADOS } \\
\text { OBTENIDOS }\end{array}$ \\
\hline $\begin{array}{l}\text { V Diplomado Regional de } \\
\text { Educación Ambiental } \\
\text { para la Sustentabilidad }\end{array}$ & $\begin{array}{lr}\text { Universidad Juárez } \\
\text { Autónoma } \\
\text { Tabasco } \\
\text { El Colegio de } \\
\text { Investigadores de } \\
\text { Tabasco }\end{array}$ & $\begin{array}{l}\text { Licenciados o egresados de } \\
\text { Biología, Ecología, Ciencias de } \\
\text { la Educación, Profesores de } \\
\text { Educación Básica y Preescolar, } \\
\text { Ingeniería Ambiental, Ingeniero } \\
\text { Agrónomo, Médico, Licenciado } \\
\text { en Derecho, así como otras } \\
\text { relacionadas con la educación } \\
\text { ambiental. Empresas con } \\
\text { Programas de Responsabilidad } \\
\text { Empresarial o que requieren } \\
\text { elaborar planes ambientales y } \\
\text { de formación y comunicación en } \\
\text { educación ambiental y } \\
\text { desarrollo sustentable. } \\
\text { Miembros de asociaciones } \\
\text { civiles ecologistas, entre otras. }\end{array}$ & 2006 & $\begin{array}{l}\text { Programa } \\
\text { Curricular } \\
\text { Antología } \\
30 \quad \text { alumnos } \\
\text { egresados } \\
\text { Simposio de } \\
\text { trabajos finales } \\
\text { Lista de } \\
\text { asistencia }\end{array}$ \\
\hline $\begin{array}{l}\text { Tercer Foro estatal de } \\
\text { educación ambiental }\end{array}$ & $\begin{array}{l}\text { SEMARNAT } \\
\text { SEDESPA } \\
\text { El Colegio de } \\
\text { Investigadores de } \\
\text { Tabasco } \\
\text { Promotora Ambiental } \\
\text { SAB de CV } \\
\text { Fundación Mundo }\end{array}$ & $\begin{array}{l}\text { Tres órdenes de gobiernos } \\
\text { Instituciones educativas } \\
\text { Universidades } \\
\text { Organizaciones empresariales } \\
\text { Organizaciones comerciales } \\
\text { Sociedad civil } \\
\text { Campesinos }\end{array}$ & 2005 & $\begin{array}{l}\text { Programa de } \\
\text { ponencias con } \\
80 \\
\text { contribuciones } \\
\text { originales } \\
\text { Conferencias } \\
\text { Magistrales } \\
\text { Memoria } \\
300 \\
\text { participantes }\end{array}$ \\
\hline
\end{tabular}




\begin{tabular}{|c|c|c|c|c|}
\hline & $\begin{array}{l}\text { Sustentable A. C. } \\
\text { Universidad Juárez } \\
\text { Autónoma } \\
\text { Tabasco } \\
\text { PROEMAC }\end{array}$ & $\begin{array}{l}\text { Indígenas } \\
\text { Público en general }\end{array}$ & & $\begin{array}{l}\text { Simposio de } \\
\text { trabajos finales } \\
\text { Lista de } \\
\text { asistencia } \\
\text { Acuerdos } \\
\text { Conclusiones } \\
\text { Directorio de } \\
\text { participantes }\end{array}$ \\
\hline $\begin{array}{l}\text { Programa estatal de } \\
\text { educación ambiental } \\
\text { Para la sustentabilidad }\end{array}$ & $\begin{array}{l}\text { SEMARNAT } \\
\text { SEDESPA } \\
\text { El Colegio de } \\
\text { Investigadores de } \\
\text { Tabasco } \\
\text { Fundación Mundo } \\
\text { Sustentable A. C. } \\
\text { Promotora Ambiental } \\
\text { SAB de CV } \\
\text { Universidad Juárez } \\
\text { Autónoma } \\
\text { Tabasco } \\
\text { PROEMAC }\end{array}$ & $\begin{array}{l}\text { SEDESPA } \\
\text { SEMARNAT }\end{array}$ & 2006 & $\begin{array}{l}\text { Programa } \\
\text { estratégico con } \\
\text { acciones y } \\
\text { cronograma de } \\
\text { operación de la } \\
\text { política de } \\
\text { educación } \\
\text { ambiental para } \\
\text { el Decenio de } \\
\text { las naciones } \\
\text { Unidas. }\end{array}$ \\
\hline Curso GLOBE & $\begin{array}{lr}\text { Universidad Juárez } \\
\text { Autónoma } \\
\text { Tabasco } \\
\text { El Colegio de } \\
\text { Investigadores de } \\
\text { Tabasco }\end{array}$ & $\begin{array}{l}\text { Profesores de Nivel } \\
\text { Licenciatura, Profesores de nivel } \\
\text { Técnico } \\
\text { Profesores de bachilleratos }\end{array}$ & 2006 & $\begin{array}{lr}\text { Actividades } \\
\text { teóricas } \\
\text { prácticas } \mathrm{y} \\
\text { base en } \mathrm{el} \\
\text { manual y } \\
\begin{array}{l}\text { prácticos } \\
\text { programa }\end{array}\end{array}$ \\
\hline $\begin{array}{l}\text { VI Diplomado regional de } \\
\text { educación ambiental } \\
\text { para la sustentabilidad }\end{array}$ & $\begin{array}{lr}\text { Universidad } & \text { Juárez } \\
\text { Autónoma } & \text { de } \\
\text { Tabasco } & \\
\end{array}$ & $\begin{array}{l}\text { Licenciados o egresados de } \\
\text { Biología, Ecología, Ciencias de } \\
\text { la Educación, Profesores de } \\
\text { Educación Básica y Preescolar, }\end{array}$ & 2008 & \\
\hline
\end{tabular}

Vol. 8 no2 


\begin{tabular}{|c|c|c|c|c|}
\hline & $\begin{array}{ll}\text { El Colegio } & \text { de } \\
\text { Investigadores } & \text { de } \\
\text { Tabasco } & \end{array}$ & $\begin{array}{l}\text { Ingeniería Ambiental, Ingeniero } \\
\text { Agrónomo, Médico, Licenciado } \\
\text { en Derecho, así como otras } \\
\text { relacionadas con la educación } \\
\text { ambiental. Empresas con } \\
\text { Programas de Responsabilidad } \\
\text { Empresarial o que requieren } \\
\text { elaborar planes ambientales y } \\
\text { de formación y comunicación en } \\
\text { educación ambiental y } \\
\text { desarrollo sustentable. } \\
\text { Miembros de asociaciones } \\
\text { civiles ecologistas, entre otras. }\end{array}$ & & \\
\hline $\begin{array}{l}\text { Curso de educación } \\
\text { ambiental para las } \\
\text { licenciaturas de ecología } \\
\text { e ingeniería ambiental }\end{array}$ & \begin{tabular}{lr} 
Plan & \multicolumn{2}{r}{ Curricular } \\
Flexible del & Modelo \\
Educativo de la \\
Universidad & Juárez \\
Autónoma & de \\
Tabasco &
\end{tabular} & & 2005 & \\
\hline $\begin{array}{l}\text { Curso de educación } \\
\text { ambiental para la } \\
\text { maestría de ciencias } \\
\text { ambientales }\end{array}$ & \begin{tabular}{lr} 
Plan & \multicolumn{2}{r}{ Curricular } \\
Flexible del & Modelo \\
de Posgrado de la \\
División & Académica \\
de & Ciencias \\
Biológicas & de la \\
Universidad & Juárez \\
Autónoma & de \\
Tabasco &
\end{tabular} & & 2005 & \\
\hline $\begin{array}{l}\text { Curso de educación } \\
\text { ambiental para el } \\
\text { doctorado de ecología y } \\
\text { manejo de sistemas } \\
\text { tropicales }\end{array}$ & \begin{tabular}{lr} 
Plan & \multicolumn{2}{r}{ Curricular } \\
Flexible del & Modelo \\
de Posgrado de la \\
División \\
de Académica \\
Biológicas de la \\
Universidad & Juárez \\
Autónoma & de \\
Tabasco &
\end{tabular} & & 2005 & \\
\hline
\end{tabular}




\begin{tabular}{|c|c|c|}
\hline $\begin{array}{lll}\text { Curso de } & \text { cultura } \\
\text { ambiental } & & \\
\end{array}$ & $\begin{array}{lr}\text { Plan } & \text { Curricular } \\
\text { Flexible del } & \text { Modelo } \\
\text { Educativo de } & \text { la } \\
\text { Universidad } & \text { Juárez } \\
\text { Autónoma } & \text { de } \\
\text { Tabasco } & \end{array}$ & 2005 \\
\hline $\begin{array}{l}\text { Maestría en Ciencias de } \\
\text { la educación ambiental } \\
\text { para la sustentabilidad }\end{array}$ & $\begin{array}{lr}\text { Universidad } & \text { Juárez } \\
\text { Autónoma } & \text { de } \\
\text { Tabasco } & \end{array}$ & 2008 \\
\hline
\end{tabular}

Fuentes: López-Hernández (2005, 2006, 2007, 2008, 2009); López-Hernández e. al. (2005, 2006, 2007).

Proyectos y actividades sobre educación ambiental para la sustentabilidad

Sobre los aspectos anteriormente considerados en las tablas I y II, los proyectos y actividades sobre educación ambiental para la sustentabilidad que realizamos o se están realizado en la universidad pública más importante de Tabasco a partir de enero de 2005 debemos destacar cuales consideramos los cinco más importantes en el ámbito no forma, y el servicio social.

1. Curso Taller composta con mujeres indígenas

2. Curso Taller PET con jóvenes indígenas

3. Curso Taller de creación artística carta de la tierra

4. Jornadas por un campus digno y limpio primera fase

5. Curso taller de cambio climático y uso y conservación del agua en escuelas del corredor GuatacalcaOxiacaque.

Por el grado de compromiso, participación, aceptación de los grupos de niños, niñas, jóvenes, señoras, padres de familia y Directores de la escuelas, y por consolidación de los proyectos de educación ambiental para el desarrollo humano de las comunidades indígenas chontales a través del Centro Holístico Mundo Sustentable de Olcuatitán Nacajuca. Con el respaldo de Mundo Sustentable, El
Colegio de Investigadores y la UJAT, además de la colaboración de CECADESU-SEMARNAT. Este modelo fue condecorado con el Premio Estatal de Ecología José N. Rovirosa por el Gobierno de Tabasco en 2008. Entregado el 5 de junio de ese año.

Los programas o actividades de educación ambiental para la sustentabilidad a nivel formal que se han impartido en el estado por personal de la UJAT durante el periodo de referencia permiten destacar diplomados, cursos, foros, documentos, escritos, publicaciones, videos, etc.).

En otro aspecto, la relación con instituciones $u$ organizaciones, ya sea gobierno, de la sociedad civil o del sector privado que contribuyen más a la realización de estas acciones son:

- Instituto Estatal de Cultura,

- El Colegio de Investigadores de Tabasco, A. C.

- Fundación Mundo Sustentable A. C.,

- Promotora Ambiental SAB de CV,

- Universidad Juárez Autónoma de Tabasco.

Grupos y sectores de mayor trascendencia a las iniciativas de la UJAT en el DEDS 
Los grupos considerados por la UJAT con los que hemos tenido mayor trascendencia a las iniciativas de nuestra institución en el DEDS se citan a continuación.

- Comunidades indígenas,

- Universidades públicas, Institutos Tecnológicos, Instituto Estatal de Cultura,

- Instituto para la Educación de los Adultos del Estado de Tabasco, específicamente la Dirección de Misiones Culturales.

- ANUIES

- Directores de División

- Sociedades de Alumnos de todas las divisiones académicas

- Gobiernos municipales

Por otra parte los sectores o grupos sociales consideramos debieran ser mejor atendidos por los programas de educación ambiental para la sustentabilidad son:

Comunidades indígenas y campesinas y sus grupos principales en el medio rural y suburbano, pues la mayoría de las acciones del sector ambiental se hacen en la ciudad capital de Tabasco con pocas repercusiones en otros municipios de Tabasco.

Las Universidades Públicas, Universidades Tecnológicas, Institutos y Bachilleratos Tecnológicos, y Tecnológicos Agropecuarios, Escuelas del Medio Rural de los municipios de la Chontalpa.

Profesores del sistema estatal, y del Instituto para la Educación de los Adultos del Estado de Tabasco (IEAT), específicamente la Dirección de Misiones Culturales.

En el estado, los grupos y organizaciones muestran un liderazgo en las acciones de educación ambiental para la sustentabilidad son:

- Universidad Juárez Autónoma de Tabasco

- División Académica de Ciencias Biológicas

- Instituto Estatal de Cultura de Tabasco

- $\quad$ El Colegio de Investigadores de Tabasco, A. C.
- Fundación Mundo Sustentable A. C.,

- Promotora Ambiental SAB de CV,

- $\quad$ Red Iberoamericana de Mercadotecnia en Salud A. C.

- Profesionales Especialistas en Medio Ambiente A. C.

- Fundación José Vasconcelos A. C.

Todas ellas han dado continuidad y congruencia a su alianza como personas y organizaciones, además han trabajado hace más de seis años conjuntamente, tanto en iniciativas de políticas públicas, organización de eventos trascendentales, fomento de proyectos sustentables, y la participación de nuevos grupos y personas. Persiguen objetivos similares que les han posibilitado la continuidad y persistencia y permanencia de sus actividades, además de sus relaciones a nivel regional, nacional e internacional.

\section{APORTES FINANCIEROS EN MATERIA DE EDUCACIÓN} AMBIENTAL

Además de las aportaciones para la sustentabilidad, otras instituciones y organizaciones que nos han apoyado presupuestalmente para las acciones referidas en las tablas I y II son:

- Fundación Mundo Sustentable A. C.

- Promotora Ambiental SAB de C. V.

- Instituto Estatal de Cultura de Tabasco (a través de fondos para acciones y proyectos de programas estatales y federales de cultura, arte y ambiente).

- Fondo Nacional Cultura y las Artes

- Fondo de Investigación UJAT

- $\quad$ Promep

Antes del inicio del DEDS, y desde hace cuatro años fortaleciendo los espacios conocidos como "Centros Holísticos" en Olcuatitán Nacajuca y Tamulté de las Sabanas, Centro que favorecen su operación, además de integrar a profesores tradicionales de las comunidades 
indígenas. Contribuyen con apoyo a la investigación científica, de educación ambiental y desarrollo de planes de negocio a nivel comunitario, otorgan becas que permiten a alumnos de nivel licenciatura, maestría y doctorado desarrollar algunas actividades de su trabajo de campo, favorecen el servicio social profesionales en las comunidades, apoyan la creación de proyectos sustentables, etc. Posibilitan alianzas estratégicas con organizaciones nacionales e internacionales, CANACINTRA, SEMARNAT, Gobierno de Tabasco, PNUMA, Banco Mundial, entre otras. Organizaciones civiles y bancarias como PVO México, y JP Morgan con fundamentales apoyos para el desarrollo de los grupos sociales de niños, jóvenes y mujeres organizados en proyectos de sustentabilidad, y en las lamentables circunstancias de las tragedias de la inundación de 2007.

Para evaluar los resultados de los programas y acciones en materia de educación ambiental para la sustentabilidad que se realizan algunas de las acciones más apropiadas para hacerlo podrían ser algunas de las que se citan a continuación:

- Visitas de supervisión

- Reuniones de diálogo con los participantes y colaboradores

- Testimonios escritos

- Memorias fotográficas

- Diarios de campo de las organizaciones involucradas

- Lista de asistencia

- Informes extensos

- Divulgación en internet y medios impresos

\section{INSTITUCIONES $Y$ ORGANIZACIONES DEL ESTADO} QUE DEBIERAN FORTALECERSE

Las instituciones y organizaciones del estado que debieran fortalecerse con la educación ambiental para la sustentabilidad son en orden de prioridad las siguientes:

- Comunidades indígenas,
- Instituto estatal de Cultura

- Universidades públicas,

- Universidades Tecnológicas,

- Institutos y bachilleratos tecnológicos, y Tecnológicos agropecuarios

- Escuelas del medio rural de los municipios.

- Profesores del sistema estatal y federal

- Profesores del Instituto para la Educación de los Adultos del Estado de Tabasco, específicamente la Dirección de Misiones Culturales.

- PEMEX, CFE, CONAGUA, CONAFOR

- Secretaria de Ecología Recursos Naturales y Protección Ambiental de Tabasco (SERNAPAM)

Temas fundamentales considerados en las acciones de educación ambiental para la sustentabilidad

Los temas más importantes que consideramos tendrían que ligarse a las acciones de educación ambiental para la sustentabilidad para tener mejores efectos en los programas que impulsa la UJAT debe tratar de dar Impulso a lo siguiente:

- $\quad$ La estrategia nacional de Educación Ambiental, así como de los programas o planes estatales en la región Sursureste.

- Profesionalización de los educadores ambientales con mejores programas institucionales y la continuidad de los mismos

- Cultura ambiental, patrimonio cultural y sustentabilidad

- Entendimiento, diálogo intercultural y cultura de paz,

- Diversidad cultural con equidad, género y ambiente

- Superación de la pobreza y alternativas agroecológicas sustentables

Vol. 8 no2

Mayo- Agosto 2009

HORIZONTE SANITARIO

62 
- Educación Ambiental, Desarrollo Humano y Promoción de comunidades saludables

Divulgación y publicación de los resultados de los proyectos exitosos

- Conocimiento, conservación, uso y Gestión de la SEMARNAT para su publicación gratuita en aprovechamiento sustentable de la biodiversidad los medios de comunicación escritos prensa, revistas a nivel estatal y/o regional

- Gestión y protección ambiental del medio

- Mejoramiento de los servicios ambientales en el medio rural,

- Consumo sustentable

- Comunicación ambiental y nuevas tecnologías de la información

- Publicaciones de capacitación

- $\quad$ Mejores presupuestos

Algunas sugerencias para darle mayor visibilidad e impacto a las acciones y programas de educación ambiental para la sustentabilidad en el estado deben de ser el fortalecimiento de las redes de trabajo académicas, civiles y políticas a nivel estatal y/o regional. Además de mejores presupuestos a nivel municipal, estatal y/o regional y a los grupos de la sociedad civil que hayan demostrado sus fortalezas y capacidades en el impulso de las estrategias.

Para fortalecer la participación de los distintos sectores sociales en los cinco años que restan al Decenio deberemos establecer como sociedades los siguientes elementos:

Entendimiento, diálogo intercultural y cultura de paz

Diversidad cultural con equidad, acerca de los campos de género y ambiente

Comunicación ambiental y nuevas tecnologías de la información

Coordinación eficiente de las instituciones del gobierno federal, estatal y municipal

Publicación gratuita en los medios electrónicos de las instituciones a nivel estatal y/o regional

Publicación de Blogs a nivel estatal y/o regional

\section{DISCUSIÓN}

En la educación ambiental no hay un solo discurso, y no puede ni debe haberlo, pues tiene una progresiva $y$ ascendente complejidad desde su nacimiento. Tal vez en el discurso oficial se está tratando de rematar a la educación ambiental, argumentando la necesidad de una institucionalización de la educación ambiental mediante las mismas recomendaciones del pasado, arguyendo posiblemente que hay una inmovilidad del paradigma, se pierden los referentes originales, y es momento hacerla más trascendente.

Una buena parte de educadores ambientales profesionales, no vemos que se esté dando una tácita aceptación, que asuma que estamos formalizando y adaptando la educación ambiental a conveniencia de los sistemas educativos oficiales. Por el contrario, la educación ambiental del presente, es una forma mucho más marginal que cultiva la evolución del campo en sus múltiples facetas.

El contexto Latinoamericano preserva los principios de una educación con una perspectiva emancipadora para lograr la sustentabilidad, sobre la base de una participación política que requieren transformarla en acciones morales, sociales y políticas con pedagogía crítica, original, participativa donde la educación ambiental deje de ser la preocupación exclusiva de unos cuantos para ponerse al servicio de la humanidad. 
La educación para el desarrollo sustentable precisaría de un modelo de instrucción que forme capacidades para el reconocimiento y aprecio del territorio donde se vive, y que permita que las representaciones sociales sobre el ambiente y la naturaleza sean parte de la vida cotidiana y contribuyan a conferirle sentido y dirección. Esta educación debe ser significativa para la reducción de las desigualdades sociales y para lograr angostar la brecha entre pobres y ricos.

No puede dejar de cuestionar los sistemas de producción, distribución y consumo existentes en el mundo, pues la dinámica económica sigue siendo la fuente de los numerosos problemas ambientales. Debemos procurar tanto la conservación de la naturaleza, como de guía para generar y fortalecer las diversas formas de aprovechamiento y restauración del patrimonio cultural y natural.

En Latinoamérica el campo a través de la educación ambiental se hacen muy evidentes los procesos no formales vinculados a distintos proyectos en las comunidades, áreas rurales, e indígenas, ante la incapacidad de las instituciones educativas oficiales de resolver los magnos problemas ambientales, en las repercusiones locales que desfavorecen el desarrollo de las personas y las sociedades hacia las oportunidades de la sustentabilidad en oposición al modelo de desarrollo actual. (López, López-Hernández y González 2007)

Coincidimos en que la producción y consumo de alimentos, la salud ambiental, el cambio climático, la diversidad cultural y natural, la vulnerabilidad y el riesgo, el consumo energético y la gestión del agua, son temas prioritarios que competen a la educación ambiental para la sustentabilidad, por ser cruciales para la vida y el desarrollo y las expectativas de una humanidad sostenible.

Se requiere que nuestra sociedad toda, asuma imperativamente, que la educación no es una sangría, sino la mejor inversión para lograr transitar hacia la sustentabilidad. Reconocemos que los avances logrados en materia de educación ambiental, si bien están manifiestos en sendos acuerdos internacionales, no están inspirando lo suficiente a la sociedad humana a contribuir al impulso transformador de nuestra relación con el planeta mediante las líneas de acción emanadas de tales acuerdos.

Las expectativas en América Latina y el Caribe, consideran que la educación ambiental siempre se ha anticipado a su asociación con este concepto al aplicarse en otros campos las alternativas que hoy adquirieren la divisa de "la sustentabilidad". El campo de la educación ambiental es un campo en disputa que, a pesar de todo ha ido cobrando una creciente relevancia.

La concepción de ambiente de acuerdo con la cartografía de corrientes(Sauvé, 2004) será útil para que la mayoría comprendan las aproximaciones de la educación ambiental al presentarnos el desarrollo de diversas estrategias, corrientes y competencias sobre "El medio ambiente naturaleza"; "El medio ambiente recurso"; "El medio ambiente problema"; "El medio ambiente medio de vida"; "El medio ambiente biosfera"; y "El medio ambiente, como proyecto comunitario"

La incorporación de la dimensión ambiental al currículum de la educación básica presenta variados grados de complejidad, a mi juicio, considera el autor uno de los más importantes es la formación de maestros, para poner en marcha propuestas, como las de transversalidad. La educación ambiental debe ser vista como la mejor inversión para el desarrollo sustentable. Es importante advertir, las opiniones sobre las experiencias en España a partir de la ley conocida como, LOGSE y sus propuestas de transversalidad, entre otras circunstancias. (LópezHernández, 2004)

Concordamos en que la educación ambiental y el desarrollo sustentable no se limitan a la incorporación de algunas asignaturas en los planes de estudio, sino que la sustentabilidad debe funcionar como un eje formativo que le confiere un matiz permanente a los currículos en su conjunto. Se reconoce que la participación social

Vol. 8 no2

Mayo- Agosto 2009

HORIZONTE SANITARIO 
responsable requiere alimentarse de un sano ejercicio del derecho a la información, por ser éste uno de los principales insumos de la corresponsabilidad. Las diversas tecnologías de la información al alcance deben utilizarse con este propósito.

No obstante, el Decenio de las Naciones Unidas de la Educación para el Desarrollo Sustentable (2005-2014) puede todavía ser una oportunidad para refrendar compromisos estableciendo alianzas con las comunidades y educadores de las naciones y sus diferentes culturas. Se trata de un momento decisorio para robustecer nuestros procesos educativos y posicionar aun más la educación ambiental y a sus actores en las políticas públicas de medio ambiente y de educación; sin embargo, se necesita más información de los motivos de las distintas agencias internacionales que han promovido el cambio del concepto de educación ambiental en los planteamientos del Decenio, ya que esto tergiversa la historia de este campo emergente y se desconocen sus contribuciones en América Latina y el Caribe a los procesos de cambio político educativos para la creación de sociedades sustentables.

El problema que está enfrentando la Educación Ambiental tiene que ver con la política, la cual enfoca sus aspectos al desarrollo económico, contrapunteándose con los que se intenta construir en el campo. Un problema de fondo, que ha tenido y tiene que ver con la toma de decisiones y el poderío económico. Debería darse una discusión significativa de lo que está pasando en los cambios que se deben tomarse en otros espacios para darle el sentido político que esta disciplina necesita hacer para influir en diversos espacios.

La educación no es un sitio con éxito relativo, pero es muy conveniente separar a la educación ambiental de otras disciplinas que educan en valores, por ejemplo, la diversidad cultural, la diversidad biológica, y considerar que las anteriores son la base de una educación basada en nuevas alternativas de compresión del avance científico sobre el ambiente, y sus bases materiales, integrando el patrimonio cultural, y la sustentabilidad, pues al estar dirigida la obra al profesorado del sistema escolar, habría que surtir de juicios, criterios y bases éticas a un sector que tendría que aprender que la educación ambiental es un campo complejo y que transita dentro de una franca relación transversal contando con elementos como son ciencia, ambiente, sociedad, cultura, política y sustentabilidad.

Se reconoce que la participación social responsable requiere alimentarse de un sano ejercicio del derecho a la información, por ser éste uno de los principales insumos de la corresponsabilidad. Las diversas tecnologías de la información al alcance deben utilizarse con este propósito.

No obstante, el Decenio de las Naciones Unidas de la Educación para el Desarrollo Sustentable (2005-2014) puede todavía ser una oportunidad para refrendar compromisos estableciendo alianzas con las comunidades y educadores de las naciones y sus diferentes culturas.

Así, después del desarrollo de la Cumbre de la Tierra de Brasil y la celebrada en Johannesburgo Sudáfrica, donde se advierten los problemas globales mundiales y sus efectos locales, debemos inculcar en los ciudadanos la importancia de lo ambiental desde los aspectos fundamentales de constituir las bases de una cultura ambiental que conlleve al desarrollo humano armónico y sustentable.

La "Declaración de Ahmedabad", implica que la EDS en un cambio desde la educación concebida como un mecanismo para proporcionar conocimiento hacia el reconocimiento de que todos y todas somos a la vez alumnos/as y maestros/as y debe estar al acceso no sólo de las escuelas sino también la sociedad civil así como de aquellos sectores tales como: empresas, ministerios, ONG, por lo cual la EDS debe ser impartida en distintos sectores de la sociedad.

Sin embargo, sobre ella, se han manifestado "observaciones $y$ vivencias personales y subjetivas" que solicitan poner interés a la EDS de acuerdo con lo acontecido en el Congreso de Educación Ambiental de Ahmedabad, India (UNESCO, UNEP, 2007), pues se considera que hay peligro ante la falta del peso latino al no haber sido consideradas 
algunas de las sugerencias y propuestas que se realizaron a la "frustrante declaración final", con base en los informes de 32 países de Europa, 19 de Asia y el Pacífico, 4 de África, uno de los Estados Unidos y dos de países como Chile y Costa Rica. Muy pobre documentación para tan importante evento. Benayas (2007)

Este colega, considera que no se aporta mucho a lo ya dicho y conocido hasta la fecha, y a pesar de originarse en un Congreso sobre Educación Ambiental a 30 años de Tbilisi no incluyó el término EA, pero si se incluyeron dos párrafos nuevos en idioma inglés a saber:

"Environmental Education processes are essential to making this transformation" $\mathrm{y}$ "Environmental Education processes support and champion Education for Sustainable Development".

Según el testimoniante, la UNESCO "sigue con la idea fija de que la Educación Ambiental ha muerto y que ahora solo se debe hablar de EDS. (Benayas, loc cit.)

\section{CONCLUSIONES}

Consideramos al decenio una buena intención debido a la importancia radicada en contribuir a la comprensión de los sucesos que posibilitarán las perspectivas de su continuidad para la formación de personas y sociedades sostenibles. Aunque tendremos que estar atentos a que esta se adapte a nuestros propios procesos y contextos y deje de ser un modelo impuesto desde el poder político de los países ricos.

Implicará constatar cuáles son las causalidades de la educación ambiental, hasta que esta pueda adquirir la capacidad de desarrollar sentidos críticos, metodológicos y pedagógicos.

Posiblemente nos posibilite ir alcanzando una humanidad con una relación con la naturaleza que nos permitiera el desarrollo de nuestras capacidades físicas e intelectuales para contribuir al desarrollo de la cultura.
Habrá que hacer la fundación de nuevas organizaciones sociales y seres humanos que influyan en el desenvolvimiento de nuevas tendencias de nuestra civilización. 


\section{REFERENCIAS BIBLIOGRÁFICAS}

- Achinelli Moira, L. 2005. Incorporación de la Educación para el Desarrollo Sustentable a la Ley de Educación Nacional. debate-educacion.educ.ar/ley/Moira20Achinelli.pdf -Similares.http://debate-

educacion.educ.ar/ley/Moira\%20Achinelli.pdf

- Benayas, J. 2007. Ahmadabad - El informe de Javier Benayas)

forotuqueque.blogspot.com/.../ahmadabad-el-informe-dejavier-benayas.html -

- Congress Environmental Education. (2005). "Decade of Education for Sustainable Development: taking it forward together", Ahmedabad, India.

- López-Hernández, E. S. 2003. Educación Ambiental. Apuntes y recomendaciones para el desarrollo sustentable. Colección José N. Rovirosa. Medio Ambiente y Desarrollo Sustentable. Universidad Juárez Autónoma de Tabasco.

- López-Hernández, E. S. y col. 2004. Juchimán, verde y oro. Programa Ambiental de la Universidad Juárez Autónoma de Tabasco para el Desarrollo Sustentable. Volumen 3. Número 3. ISSN 1665-326232-48. : 32-50

- López-Hernández, E. S.; M. T. Bravo M; E. González G. 2005. La profesionalización de los educadores ambientales hacia el desarrollo humano sustentable. ANUIES. Biblioteca de la Educación Superior. Universidad Juárez Autónoma de Tabasco.

- López-Hernández, E. S. y Rodríguez Luna, A. R. 2005. Programa Estatal de Educación Capacitación y Comunicación Ambiental del estado de Tabasco. En: LópezHernández, E. S.; M. T. Bravo M; E. González (coords). 2005. La profesionalización de los educadores ambientales hacia el desarrollo humano sustentable. ANUIES. Biblioteca de la Educación Superior. Universidad Juárez Autónoma de Tabasco.
- López-Hernández, E. S. y E. González G. 2005. Educación Ambiental para el Desarrollo Sustentable. Diez años para cambiar el Mundo. Editorial. Horizonte Sanitario. Volumen 4 Número 1. ISSN 1665-326. : 4-8

- López-Hernández, E. S. 2005. Jornadas Universitarias por un Campus digno y limpio. Horizonte Sanitario. Volumen 4 Número 3. ISSN 1665-3262. : 35-40

- López-Hernández, E. S. y Carlos López R. 2006. Aprendizajes y observaciones globales en beneficio del medio ambiente. El programa Globe en Tabasco, jornadas. Horizonte Sanitario. Volumen 5. Número 2. ISSN 1665-3262. : 55-65.

- López-Hernández, E. S. y Priego Alvarez H. 2007. Aprendizajes en la construcción del plan ambiental de una Universidad pública del trópico húmedo. "Juchimán Verde y Oro. Horizonte Sanitario. Volumen 6. Número 1. Nillahermosa, Tabasco, México/ISSN 1665-3262: 8-29

- López-Hernández, E. S. 2009. Maestría en Ciencias de la Educación Ambiental. Blog de Profesores Investigadores. http://maestriaencienciaseducacionambiental.blogspot.com/ - Rydén L. (2003). "Education for Sustainable Developmentexperiences from de Baltic 21 Education project", Report from the Nordic Council of Minister's seminar held in Karlskona on 12-13 June 2003, Sweden.

- Sauvé, L. 2004. Una cartografía de corrientes en educación ambiental. In: Sato, M, Carvalho, I. (Orgs). A pesquisa em educação ambiental: cartografias de uma identidade narrativa em formação. Porto Alegre: Artmed.

- Secretaría de Medio Ambiente y Recursos Naturales. 2005. Compromiso Nacional por la década de la educación para el decenio de las Naciones Unidas de la educación para el desarrollo

sustentable.www.semarnat.gob.mx/educacionambiental/.../c ompromiso_nacional.pdf 
- UNESCO, UNEP y Gobierno de la India. (2007)

Declaración de Ahmedabad.

http://www.tbilisiplus30.org/Declaration20_spanish.pdf

- World Comission on Environment and Development (1987).

"Our Common Future", Oxford.

Vol. 8 no2

Mayo- Agosto 2009

HORIZONTE SANITARIO

68 